\title{
Acute ischemic stroke in a patient with gastrointestinal complaints of COVID-19 infection
}

\author{
Oana Obrisca ${ }^{1}$, Anca A. Arbune ${ }^{1}$, Mirela Draghici ${ }^{1}$, Eugenia Irene Davidescu ${ }^{2,3}$, \\ Ayghiul Mujdaba-Elmi², Adriana O. Dulamea ${ }^{1,3}$ \\ ${ }^{1}$ Neurology Clinic, Fundeni Clinical Institute, Bucharest, Romania \\ ${ }^{2}$ Neurology Clinic, Colentina Clinical Hospital, Bucharest, Romania \\ 3"Carol Davila" University of Medicine and Pharmacy, Bucharest, Romania
}

\begin{abstract}
The most important pandemic in the last century triggered by SARS-CoV-2 infection is ongoing, and longterm implications are still unknown. We report the case of a 65-year old man, who presented with sudden onset of right-sided limb weakness, facial asymmetry, and dysarthria, accompanied by vomiting and headache. The initial neurological exam suggested acute stroke, although the cerebral CT-scan was not relevant neither recent ischemic, nor haemorrhage findings. According to the therapeutic protocol, intravenous thrombolytic therapy was timely administered, with a neurofluctuating course. The infusion was premature interrupted due to the sudden hematemesis, following by concurrent diarrhoea, abdominal cramps and recurrent vomiting. The new cerebral imaging confirmed the acute ischemic stroke in the vertebrobasilar territory and the abdominal CT-exam displayed an inflammatory aspect inflammation surrounding the right kidney and the proximal colon, minimal right pleural effusion and hypo-ventilated areas in the pulmonary bases. The laboratory work-up revealed mild leukocytosis with neutrophilia, increased levels of D-dimers, altered renal function. Considering the epidemic COVID-19, a RT-PCR test for SARS-CoV-2 have been request and, surprisingly, it was positive. Previous unknown paroxysmal atrial fibrillation was found at admission by electrocardiography, suggesting the cardioembolic nature of the ischemic stroke, while a favouring role of infection is possible. The subsequent evolution was positive, following by the secondary prevention with oral anticoagulation, along with hypotensive and statin medication. Apart from the respiratory affection, COVID-19 infection can be present in patients with other major medical emergencies, including acute stroke, with no apparent sign of infection. Additional signs of gastrointestinal affection, along with attentive evaluation of abdominal CT-scan represent important hints for concomitant SARS-CoV-2 infection.
\end{abstract}

Keywords: COVID-19, ischemic stroke, thrombolysis, diarrhoea

\author{
Abbreviations \\ ACE2 Angiotensin converting enzyme 2 \\ ALT Alanin-aminotranspherase \\ AST Aspartate-aminotranspherasa \\ ASPECTS The Alberta Stroke Programme Early CT Score \\ CK Creatinkinase
}

\section{INTRODUCTION}

The emergency of the new coronavirus SARS$\mathrm{CoV}-2$ triggered the most important pandemic in the last century that is ongoing, and long-term implica-
CK-MB Miocardial fraction of CK

NIHSS The National Institutes of Health Stroke Scale SOFA Sequential organ failure assessment RT-PCR Reverse-transcriptase-polymerase-chain-reaction NRV Normal reference values named COVID-19. The majority of patients suffer from a mild disease, $20 \%$ require hospital admission with severe disease accompanied by respiratory dis- 
tress and $5 \%$ of them progress to critical forms, with septic shock, multiple organ failure and major coagulation disorders, which involve intensive care management (1).

People over 60 years-old and with several comorbidities, as arterial hypertension, coronary artery disease, diabetes, smoking, obesity, have greater odds for in-hospital death $(2,3)$. Furthermore, other predictors of death have been identified, such as higher SOFA scores and D-dimer values over $1 \mu \mathrm{g} / \mathrm{ml}$, high levels of serum interleukin-6, troponin-I, lactate dehydrogenase (LDH) and ferritin, as well as marked lymphopenia (2-4). Other studies confirmed the previous observations that higher serum leukocyte, neutrophil and C-reactive protein levels correlate with a more severe disease (5-7).

COVID-19 infection is a systemic disease. The most common symptoms are fever, cough, short breath, chills, muscle pains, headache, throat pain, loss of taste or smell. Oftentimes, neurological manifestations can precede the typical features and are related either to central nervous system affection (e.g. vertigo, headache, impaired consciousness, stroke, acute transverse myelitis), or to peripheral nervous system involvement (taste and smell impairment, Guillain-Barré syndrome, neuropathic pain) $(2,8,9)$.

The gastrointestinal symptoms, as diarrhoea, anorexia, vomiting, abdominal pains, are reported in less than $10 \%$ of cases $(10)$.

Other clinical presentations that are rare reported are dermatologic, cardiovascular or vasculitis manifestations. As studies progress toward the elucidating the pathogenic mechanisms of SARS-CoV-2, we will be able to better understand the influence of this acute infection on the evolution of chronic pathologies.

\section{CASE PRESENTATION}

We report the case of a 65-year-old non-smoker man, without any significant comorbidities, who presented to the neurology clinic for right-sided limb weakness, facial asymmetry and speech difficulties, symptoms that had occurred suddenly, 2 hours and 40 minutes prior to admission. He did not follow any permanent treatment at home. On the ambulance, he presented nausea and vomiting, associated with mild headache. At admission, the general examination revealed a somnolent patient, with elevated blood pressure of $180 / 100 \mathrm{mmHg}$, irregular pulse of $75 / \mathrm{min}$, oxygen saturation of $97 \%$, no carotid/cardiac bruits, normal pulmonary and abdominal examination, preserved diuresis. The neurological examination showed marked dysarthria, divergent strabismus of the left eye, anisocoria, right facial palsy, right hemiplegia, right Babinski sign, no sensory deficits or ataxia, summing up an initial NIHSS score of 14. The first electrocardiogram confirmed paroxysmal atrial fibrillation. The initial cerebral CT scan did not show any signs of early ischemia, nor haemorrhage and ASPECTS score of 10 points.

The presumptive diagnosis was acute ischemic stroke in the vertebrobasilar territory. According to the protocol for the management of stroke, after verifying the eligibility criteria allowed the decision to administer the intravenous thrombolytic therapy (Alteplase $0.9 \mathrm{mg} / \mathrm{kg}$ ) at about 3 and a half hours after stroke onset (11).

The neurological symptoms did fluctuate during the first part of thrombolysis, as impressive recovery of the motor deficit had alternated with profuse somnolence and right-sided hemiplegia. The therapy with Alteplase was discontinued when $75 \%$ of the dose was infused, due to the occurrence of sudden hematemesis, followed by recurrent vomiting, concurrent diarrhoea and abdominal cramps, which contributed to a marked psychomotor agitation and restlessness.

The repeated brain CT scan at 24-hours after thrombolysis revealed a recent hypodensity located in the left superior cerebellar hemisphere, suggesting recent ischemic stroke in the territory of the left superior cerebellar artery (SCA) (figure 1).

In addition, the patient underwent abdominal CTexam, which displayed an inflammatory aspect of the proximal colon, with no signs of peritonitis/ascites, as well as minimal right pleural effusion and hypo- ventilated areas in the pulmonary bases (figure 2).

The laboratory work-up consisted leukocytosis with neutrophyl/lymphocyte rate of 10.53 increasing to 364.6 in 24 hours. The inflammation markers (fibrinogen, C-reactive protein) and the levels of ALT, AST, CK and CK-MB were normal, while we found abnormal high values of LDH (1,2 x NRV), serum creatinine (eGRF $57 \mathrm{ml} / \mathrm{min} / 1.73 \mathrm{~m}^{2}$ ) and D-dimers of $9.89 \mu \mathrm{g} / \mathrm{ml}$. Moreover, in the epidemic context of the COVID-19, the screening test of the nasopharyngeal swabs by RT-PCR assay was positive for SARSCoV-2 and implied the re-evaluation of the case and an extensive epidemiological investigation. 

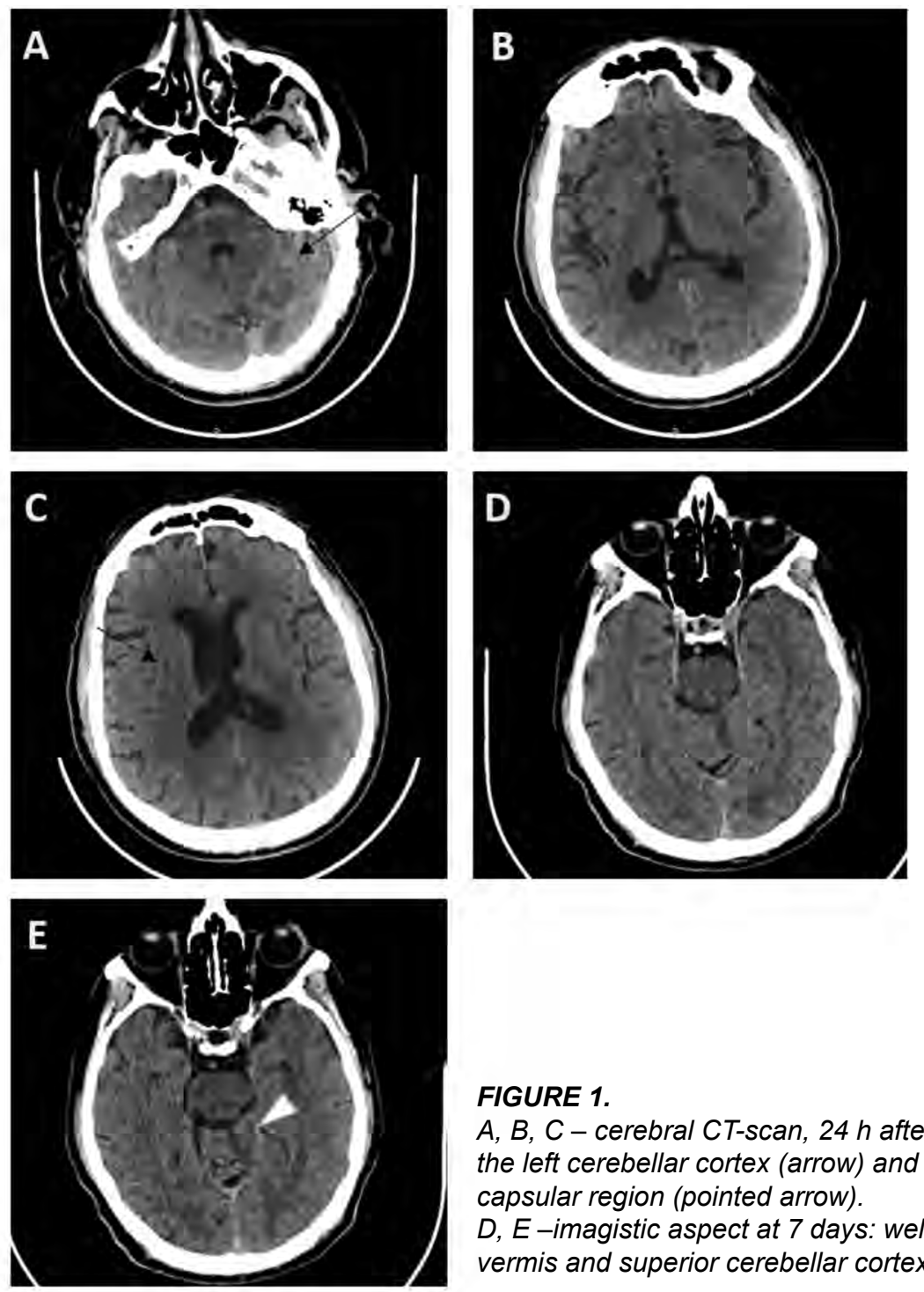

FIGURE 1.

A, B, C-cerebral CT-scan, $24 \mathrm{~h}$ after thrombolysis: note the recent hypodensity in the left cerebellar cortex (arrow) and right chronic lacunar infarct in the lenticulocapsular region (pointed arrow).

$D, E$-imagistic aspect at 7 days: well-delineated cerebral infarct, involving the left vermis and superior cerebellar cortex (SCA territory) (arrowheads).

The patient was transferred to a COVID-19 dedicated unit and he benefited of several clinical investigations that have been done that excluded symptomatic large-vessel-disease, significant valvular heart diseases or intracardiac thrombi or vegetations. The neurological and digestive symptoms improved significantly in the following days. Despite the reserved prognosis, the patient was discharged virologic cured ( 2 consecutive samples negative by RT-PCR-SARS$\mathrm{CoV}-2$ ), with normal respiratory and cardiac function, none gastrointestinal symptom and significant neurological improvement, although mild right ataxic hemiparesis and dysarthria persisted (modified Rankin score of 2 points). Secondary preventive oral anticoagulation with apixaban $5 \mathrm{mg}$ x $2 /$ day, along with antihypertensive medication and statins were recommended after discharge.

\section{DISCUSSIONS}

The analysis of this case was justified by the clinical peculiarities (table 1).

The pathogenesis of the new SARS-CoV-2 is explained by the affinity for angiotensin converting enzyme 2 (ACE2) receptors of the cells subphase, followed by binding to receptors, cells entry and cytopathic effect (12).

High density of ACE2 receptors on the pulmonary alveolar cells and macrophages are related to the predominance of respiratory symptoms, although ACE receptors are also found on endothelial cells, with systemic distribution that involve the vasculitic and coagulation damages (1). Experimental data have evidenced other potential targets of the virus, due to the presence of ACE2 on variate cells, as monocytes, 


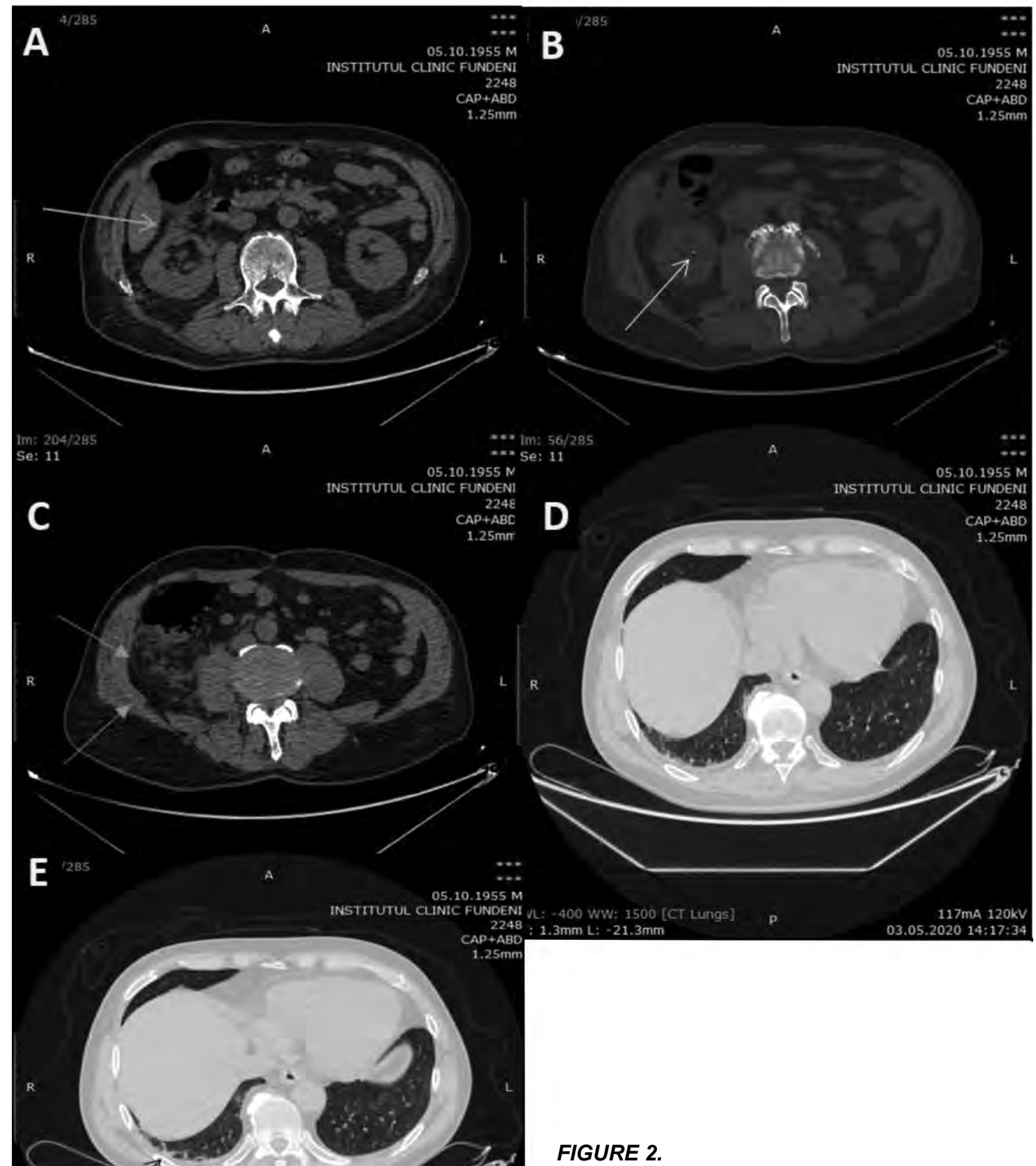

$A, B$ - abdominal CT-scan showing thickened aspect of the renal fascia, densification of perirenal fat and renal microcalculations (arrows).

$C-$ pericolic fluid and fat strandings (arrows).

$118 \mathrm{~mA} 120 \mathrm{kV}$
$202014: 17: 34$

$D, E$ - CT-scan, aspect of the lung bases, note the basal hypoventilated area (arrows).

myocardial cells, epithelial cells of the distal tubes of the kidney, other cells of testis, hypophysis, parathyroid glands, suprarenal cortex, sebaceous glands, as the epithelial cells of the digestive tract and pancreatic acinar cells (12).

The increased inflammatory response to the viral infection is believed to be secondary to accelerated production of type 2 cytokines. The inflammation of endothelium is the consequence of cytokine overpro- duction as well as cytopathic direct effect of the virus $(2,4)$. Association of impaired function of T- and Bcells in the elderly and increased levels of procoagulant factors are able to induce hemodynamic alterations (4,5). In consequence, the SARS-CoV-2 infection determines multiple organ damage through hyperinflammatory reaction (6).

Although the defining clinical criteria of COVID-19 do not include digestive symptoms, many confirmed 
TABLE 1. The peculiarities of a clinical case with COVID-19 and acute stroke

1. The inf COVID-19 was atypical, due to the absence of characteris 7 ymptoms as fever, respiratory disorders, loose of taste or smell sense, either at the beginning or during the ev of the disease.

2. Acute ischemic stroke was inaugural for COVID-19 in an elderly pa $t$ without apparent $c \quad$ and was incompletely thrombolysed, due to a haemorrhagic event.

3. Upper diges e? haemorrhage occurred in the context of thrombolysis and was followed by clinical and imaging manifesta ? ? COVID-19 inf ?that is reported by the medical literature in less than $10 \%$ of cases.

4. The absence of clinical and epidemiological criteria of inf led to the pa t's hospitaliza in a non-COVID unit, with consequences on the occupa risk of medical sta and on the ther inf thrombolysis.

5. The decision of thrombolysis before obtaining the result of RT-PCR-CoV-2 test was required by the inside the "ther ?window", while there was no suspicion of COVID-19, based on clinical and epidemiological data.

patients have reported some derangements during the illness course, related to the lesions of intestinal epithelium, abdominal vascular damages or side effects of some medication, usually concurrent with respiratory symptoms (14). The most common digestive manifestations of COVID-19 are diarrhoea, nausea or vomiting and abdominal pain, with higher rates in Caucasians, most occurring in association with respiratory symptoms. Isolated abdominal pain is the rarest manifestation $(13,14)$. A few studies have underlined the importance of abdominal imaging in cases with signs of digestive tract affliction to observe the lung bases in order to search for typical SARS-CoV-2 lesions $(14,15)$. This position is confirmed in the present case, as the abdominal CT revealed minor right pleurisy and basal bilateral hypo-ventilated areas, although respiratory symptoms were irrelevant. There are only few data on abdominal imaging related to COVID-19. A published study reported that the inflammation of the bowel walls and cholestasis are the main results, similar to the finding in our case (16).

The neurologic injuries related to COVID-19 involve complex pathogenic mechanisms, as neuronal lesion by direct viral neuroinvasion, ischemia by cerebrovascular hypoxia, inflammation and hypercoagulability (17).

The common neurologic symptoms related to COVID-19 indicate the affection of both central and peripheral nervous system and the most frequent complaints are dizziness, headache, taste and smell impairment (5). One study indicates associations between
CNS symptoms and lower lymphocyte and platelet counts as well as higher serum urea nitrogen (5). Neurological manifestations of COVID-19 during the severe infection is associated with more frequent neurological involvement such as acute ischemic and haemorrhagic stroke, cerebral venous thrombosis, encephalopathy, as well as psychomotor restlessness or delirium $(5,6,9)$. According to a Chinese study, 13 of 221 hospitalised patients with COVID-19 developed acute strokes, with an estimated risk of $4.9 \%$ (18).

Most reports indicate the emergence of ischemic cerebral thromboembolic events later in the course of the disease $(19,20)$. Interestingly, some authors point out that neurological manifestations in severe cases tend to occur early in the course of the infection, with many reported stroke cases without typical infection symptoms at presentation (5). The patients usually developed respiratory distress and characteristic lung lesions later on during admission (5). A particular case series reports large vessel ischemic stroke in younger patients, without previously known cardiovascular comorbidities, suggesting a potential link to the collateral SARS-CoV-2 infection (19,21).

COVID-19 is not a contraindication for the eligible patients with ischaemic stroke. However, supplementary precautions of the balance benefit/ risk evaluation are required and special procedures for infection prevention must be considered during the medical care in COVID-19 dedicated units (22).

Atrial fibrillation was considered the most probable cause of the cerebrovascular event, while COVID-19 could be an additional factor by infectious hypercoagulability.

The decision for thrombolysis in the present case was influenced by ethical considerations for the patient benefit, balancing the chance of the therapy with the best prognosis and the low probability of COVID-19 infection.

\section{CONCLUSION}

High risk of acute stroke is related to COVID-19 infection, mostly in the older people with some comorbidities. During COVID-19 epidemic, the major suspicion of infection should be considered in patients with acute strokes, considering the possible atypical cases and the absence of respiratory symptoms. More frequent abdominal CT imaging should be recommended to improve the diagnostic of gastrointestinal findings of COVID-19 infection. 


\section{REFERENCES}

1. Cevik M, Bamford C, Ho A. COVID-19 pandemic - A focused review for clinicians. Clin Microbiol Infect. 2020.

2. Mao L, Jin H, Wang $M$ et al. Neurologic Manifestations of Hospitalized Patients with Coronavirus Disease 2019 in Wuhan, China. JAMA Neurol. 2020:1-8.

3. Zhou F, Yu T, Du R, et al. Clinical course and risk factors for mortality of adult inpatients with COVID-19 in Wuhan, China: A retrospective cohort study. Lancet. 2020.

4. Ruan $Q$, Yang K, Wang W, Jiang L, Song J. Clinical predictors of mortality due to COVID-19 based on an analysis of data of 150 patients from Wuhan, China. Intensive Care Med. 2020.

5. Mehta P, McAuley DF, Brown M, Sanchez E, Tattersall RS, Manson JJ. COVID-19: Consider cytokine storm syndromes and immunosuppression. Lancet. 2020;395(10229):1033-1034.

6. Cots JM, Alós JI, Bárcena M, Boleda X, Cañada JL, Gómez N, Mendoza A, Vilaseca I, Llor C. Recommendations for Management of Acute Pharyngitis in Adults. Acta Otorrinolaringologica (English Edition). 2015;66(3):159-70.

7. Chen $G, W u D$, Guo $W$ et al. Clinical and immunologic features in severe and moderate Coronavirus Disease 2019. J Clin Invest. 2020.

8. Ahmad I, Rathore FA. Neurological Manifestations and Complications of COVID-19: A Literature Review. J Clin Neurosci. 2020.

9. Helms J, Kremer S, Merdji $\mathrm{H}$ et al. Neurologic Features in Severe SARS-CoV-2 Infection. N Engl J Med. 2020:1-2.

10. Cheung KS, Hung IF, Chan PP, et al. Gastrointestinal Manifestations of SARS-CoV-2 Infection and Virus Load in Fecal Samples from the Hong Kong Cohort and Systematic Review and Meta-analysis [published online ahead of print, $2020 \mathrm{Apr} 3$ ]. Gastroenterology. 2020;S0016-5085(20)30448-0.

11. Monitorul Oficial Partea I, nr. 34bis/11.I.2019. Anexa la Ordinul ministrului sănătătii $\mathrm{nr}$. 17/2019 privind aprobarea protocolului pentru tratament intervenţional al pacienţilor cu accident vascular cerebral acut, din 07.01.2019 - 3-60. http://www.cnas.ro/castr/media/ postFiles/Monitorul\%20Oficial\%20nr\%2034bis_11.01.2019.pdf.

12. Zhou F, Yu T, Du R et al. Clinical course and risk factors for mortality of adult inpatients with COVID-19 in Wuhan, China: A retrospective cohort study. Lancet. 2020;395(10229):1054-1062.

13. Wong SH, Lui RNS, Sung JJY. COVID-19 and the digestive system. J Gastroenterol Hepatol. 2020.

14. Dane B, Brusca-Augello G, Kim D, Katz DS. Unexpected Findings of Coronavirus. AJR. 2020:215:1-4.

15. Siegel A, Chang PJ, Jarou ZJ, et al. Lung Base Findings of Coronavirus Disease (COVID-19) on Abdominal CT in Patients With Predominant Gastrointestinal Symptoms. Am J Roentgenol. 2020.

16. Bhayana R, Som A, Li MD et al. Abdominal Imaging Findings in COVID-19: Preliminary Observations. Radiology. 2020;201908.

17. Tunç A, Ünlübaş Y, Alemdar M, Akyüz E. Coexistence of COVID-19 and acute ischemic stroke report of four cases. J Clin Neurosci. 2020;S0967-5868(20)31081-X.

18. Li Y, Wang M, Zhou Y et al. Acute cerebrovascular disease following COVID-19: A single center, retrospective, observational study. SSRN Electronic Journal 2020.

19. Oxley TJ, Mocco J, Majidi S et al. Large-Vessel Stroke as a Presenting Feature of COVID-19 in the Young. N Engl J Med. 2020.

20. Valderrama EV, Humbert K, Lord A, Frontera J, Yaghi S. Severe Acute Respiratory Syndrome Coronavirus 2 Infection and Ischemic Stroke. Stroke. 2020.

21. Qureshi Al, Abd-Allah F, Al-Senani F, Jani V et al. Management of acute ischemic stroke in patients with COVID-19 infection: Report of an international panel. International Journal of Stroke 2020.

22. Baracchini $C$, Pieroni A, Viaro $F$ et al. Acute stroke management pathway during Coronavirus-19 pandemic. Neurol Sci. 2020; 41:1003-1005.

Conflict of interest: none declared Financial support: none declared 